\title{
MYL9 promotes nasopharyngeal carcinoma carcinogenesis, progression and paclitaxel resistance by regulating EMT signals
}

Rong Li ( $\sim$ nflirong@163.com )

Southern Medical University

Ruifang Zeng

Southern Medical University

Hanyu Wang

Sun Yat-sen University Cancer Center

Yunhui Qiu

Southern Medical University

Luosheng Zhang

Southern Medical University

\section{Research Article}

Keywords: MYL9, Poorly differential nasopharyngeal squamous carcinoma, carcinogenesis, progression, paclitaxel resistance

Posted Date: February 22nd, 2022

DOI: https://doi.org/10.21203/rs.3.rs-1217308/v1

License: (c) (1) This work is licensed under a Creative Commons Attribution 4.0 International License. Read Full License 


\section{Abstract}

Background: More than $40 \%$ of nasopharyngeal carcinoma (NPC) come from China, and the incidence rate in South China is extremely high. Poorly differential nasopharyngeal squamous carcinoma (PDNSC) is the most common pathological type, while the detailed molecular mechanisms remain unclear.

Objectives: This study aimed to explore the role of MYL9 in the carcinogenesis, progression and chemoresistance of PDNSC, as well as the specific molecular biological mechanisms of pathogenesis and paclitaxel resistance, and to provide a new theoretical basis of targeted drugs for PDNSC.

Methods: In this study, Reverse transcription-quantitative polymerase chain reaction (RT-qPCR) and Western blot were performed to detect the mRNA and protein expression of MYL9 in PDNSC tissues and cell lines (HONE-1 and SUNE-1), respectively. Moreover, MTT and EdU assays were performed to detect the proliferation function of MYL9 in PDNSC cells; Transwell and Boyden assays were performed to detect the migration and invasion function of MYL9; The $\mathrm{IC}_{50}$ value of paclitaxel was performed to detect the effect of MYL9 on drug sensitivity in PDNSC cells. In addition, Western blot assay was performed to detect the mechanism of MYL9 in PDNSC cells.

Results: The results showed that MYL9 mRNA was much higher in PDNSC tissues compared with that in paratumor tissues, and MYL9 knockdown suppressed the proliferation, migration and invasion, while reducing the $\mathrm{IC}_{50}$ value of paclitaxel, in PDNSC cells. Mechanistic study showed that MYL9 knockdown inhibits the epithelial-mesenchymal transition (EMT) signals and downstream chemoresistant factors including $A B C G 2$ and $A B C B 1$.

Conclusion: MYL9 is upregulated in PDNSC, and it promotes PDNSC carcinogenesis, progression and paclitaxel resistance via regulating EMT signals and downstream chemoresistant factors, and it may be used as a useful patent of therapeutic target in PDNSC.

\section{Introduction}

Nasopharyngeal cancer (NPC) is actually a "rare disease" globally. About 1.2 out of every 100,000 people will develop this cancer ${ }^{[1-3]}$, and approximately $90 \%$ cases are poorly differential nasopharyngeal squamous carcinoma (PDNSC) ${ }^{[1,4]}$. Persistent infection with high-risk Epstein-Barr virus (EBV) is a primary factor which causes NPC ${ }^{[5]}$. Although the molecular mechanisms of NPC initiation and progression in addition to EBV infection have been widely explored ${ }^{[1,2,5]}$, the detailed molecular mechanisms remain unclear. Therefore, it is very important to explore the detailed mechanisms driving PDNSC carcinogenesis and progression, which may help to find effective targeted treatment strategies and reduce the morbidity and mortality of PDNSC.

Myosin regulatory light chain 9 (MYL9), also known as myosin regulatory light chain gene, which is located on human chromosome 20q11.23. It contains 4 exons and encodes 172 amino acids. MYL9 plays crucial function in various tumor progression and metastasis ${ }^{[6-12]}$. Recently, several reports show 
that MYL9 expression is different in various human tissues. In bladder, colon, non-small cell lung, gastric, prostate cancers ${ }^{[13-16]}$, total MYL9 expression is lower compared with the corresponding normal tissue. Conversely, MYL9 overexpression is associated with adverse clinical outcomes in esophageal squamous cell carcinoma ${ }^{[7]}$ and glioblastoma ${ }^{[10]}$. However, to the best of our knowledge, there has been no research between MYL9 and PDNSC yet.

The main treatment for NPC is radiotherapy. For patients with advanced stages, combined paclitaxel chemotherapy is usually required, with a cure rate of $70-85 \%{ }^{[17]}$. However, $20-30 \%$ of metastatic patients usually develop resistance after 6 courses of paclitaxel chemotherapy ${ }^{[18-20]}$. Current research ${ }^{[21-24]}$ believes that various biological, chemical and other factors that lead to tumor cell drug efflux, inactivation or inability of drugs to reach the tumor site and increased DNA repair can all lead to paclitaxel resistance. Nevertheless, the molecular biological mechanism of paclitaxel resistance in NPC remains unclear.

The present study identified that MYL9 is upregulated in PDNSC, and serve as an oncogenic role which regulated PDNSC carcinogenesis, progression and paclitaxel chemoresistance via EMT signals and its downstream chemoresistant factors, and it may be a useful patent of treatment target in PDNSC.

\section{Methods}

\section{Cells and tissues}

Human nasopharyngeal poorly differentiated squamous cell carcinoma cell line (SUNE-1 and HONE-1) (EBV-positive) were obtained from Cell Resource Center, Shanghai Institute of Life Sciences, Chinese Academy of Sciences, which was maintained in RPMI-1640 (Gibco) supplemented with 10\% fetal bovine serum (FBS, Hyclone) at $37^{\circ} \mathrm{C}$ with $5 \% \mathrm{CO}_{2}$. Moreover, 20 pairs of PDNSC and paratumor tissues were collected from patients undergoing a surgical procedure at the Cancer Center, Integrated Hospital of Traditional Chinese Medicine, Southern Medical University. These patients provided written informed consent prior to the operation. This study was approved by the Ethics Committee of the Integrated Hospital of Traditional Chinese Medicine, Southern Medical University.

\section{Reverse transcription-quantitative polymerase chain reaction (RT-qPCR)}

RNAs from clinical samples or cells were subjected to detect the mRNA expression of MYL9 mRNA using RT-qPCR analysis ${ }^{[25]}$. SuperScript III RT (Invitrogen; Thermo Fisher Scientific, Inc.) was used to synthesize complementary DNA from $1 \mu \mathrm{g}$ of total RNA. The PCR mixture contained $1 \mu \mathrm{L}$ of the cDNA templates, $1 \times$ final concentration of SYBR Green (Toyobo, Osaka, Japan), and the specific primers. The sequences of the primers synthesized by IGE Co., Ltd (Guangzhou, China). All tests were conducted in triplicate on a LightCycler 480 II (Roche, Basel, Switzerland). Relative expression of MYL9 mRNA were calculated 
with $2^{-\Delta \Delta C t}$ method ${ }^{[25]}$. The specific primer ${ }^{[26]}$ sequences were as follows: MYL9 forwards, $5^{\prime}$ AGTTTGTCTCGGAGCTGTGG-3' and reverse, 5'-GGTTCGTGTTCCTCAGCGTA-3'; ABCB1 forwards, 5'GAGGAAGACATGACCAGGTATGC-3' and reverse, 5'-AGCTGCCAGGCACCAAAATG-3'; GAPDH forwards, 5'CCATCTTCCAGGAGCGAGAT-3' and reverse, 5'-TGCTGATGATCTTGAGGCTG-3'.

\section{Transfection}

The siRNA targeting MYL9 (siMYL9) and negative control (siNC) were purchased from Guangzhou IGE Co., Ltd. (Guangzhou, China). The siMYL9 sequences was 5'-GCAACATCGTCTTCAAGAA-3'. siRNAs (including siMYL9 and siNC group) were transfected into SUNE-1 and HONE-1 cells using Lipofectamine ${ }^{\circledR}$ 2000 (Invitrogen; Thermo Fisher Scientific, Inc., Waltham, MA, USA) according to the manufacturer's protocol ${ }^{[25]}$, respectively. This assay was performed as described in a previous study ${ }^{[25]}$.

\section{MTT assay}

MTT was used to detect the effect of MYL9 expression on PDNSC cell viability at different time points (1, 2, 3 and 4 days after siMYL9 transfection). This assay was performed as described in a previous study ${ }^{[25]}$.

\section{EdU analysis}

Proliferating SUNE-1 and HONE-1 cells (including siMYL9 and siNC group) were examined using the CellLight EDU Apollo 488 or 567 In Vitro Imaging kit (Guangzhou Ribobio Co., Ltd.) according to the manufacturers' protocols, respectively. This assay was performed as described in a previous study ${ }^{[25]}$.

\section{Transwell assay}

Cell migration assay was performed using the Transwell chambers $(8 \mu \mathrm{m}, 24-w e l l$ insert; Corning Inc., Lowell, MA, USA). Briefly, cells ( $\left.1 \times 10^{5}\right)$ in serum-free RPMI- 1640 medium were added to the upper chamber, and medium containing FBS was added to the lower chamber. Cells were incubated for $12 \mathrm{~h}$. Finally, the cells which migrated into the lower chambers were fixed with methanol, stained with crystal violet and counted in six random fields ${ }^{[25]}$.

\section{Boyden assay}

Cell invasion assays was performed using the Transwell chambers $(8 \mu \mathrm{m}, 24$-well insert; Corning Inc., Lowell, MA, USA). For the invasion assay, diluted Matrigel (BD Biosciences) was used to pre-coat the insert membrane. $1 \times 10^{5}$ cells were cultured for $36 \mathrm{~h}$ under the same conditions. Finally, the cells which 
invaded into the lower chambers were fixed with methanol, stained with crystal violet and counted in six random fields ${ }^{[25]}$.

\section{Drug sensitivity test in vitro}

Drug sensitivity test was determined by the MTT assay ${ }^{[26]}$. Cells were seeded in 96 -well plates at a density of $2 \times 10^{3}$ cells/well and treated with $0,1,2,4,8 \mu \mathrm{M}$ paclitaxel (No: 33069-62-4, Shanghai yuanye Bio-Technology Co., Ltd) for $48 \mathrm{~h}$. Subsequently, $20 \mu \mathrm{L}$ of MTT ( $5 \mathrm{mg} / \mathrm{ml}$; Sigma-Aldrich) was added to each well and incubated at $37^{\circ} \mathrm{C}$ for $4 \mathrm{~h}$. Then supernatants were removed and $150 \mu \mathrm{L}$ of DMSO (SigmaAldrich) was added to measure the absorbance value (OD) of each well at $490 \mathrm{~nm}$. The calculated rates were used for curve fitting and calculation of IC50. Experiments were performed three times.

\section{Western blot}

The SUNE-1 and HONE-1 cells were washed three times with ice-cold PBS and total protein was harvested using RIPA lysis buffer (Beyotime Institute of Biotechnology) containing PMSF (Bio-Rad Laboratories, Inc.) and Phosphatase inhibitors (Bio-Rad Laboratories, Inc.) (100:1:1). This assay was performed as described in a previous study ${ }^{[25]}$. The primary antibodies included MYL9 (15354-1-AP, Proteintech, Rabbit), N-cadherin (66219-1-Ig, Proteintech, Mouse), E-cadherin (60335-1-Ig, Proteintech, Mouse), Vimentin (10366-1-AP, Proteintech, Rabbit), ABCB1 (22336-1-AP, Proteintech, Rabbit), ABCG2 (27286-1-AP, Proteintech, Rabbit), and GAPDH (CW0100M, CWBIO, Mouse). The following secondary antibodies were used: HRP-conjugated goat anti-rabbit (cat. no. 6401-05; dilution 1:5000; Biovision, Inc.) and HRPconjugated anti-mouse IgG antibody (cat. no. 6402-05; dilution 1:5000; Biovision, Inc.).

\section{Statistical analysis}

Each experiment was performed at least three times. The data were presented as the mean \pm SD of more than three independent experiments. The significance between two groups were analyzed by Student's ttest. Graphs were made using GraphPad Prism 7 (GraphPad Software, Inc.) software. All of $p<0.05$ were considered as statistically significant.

\section{Results}

\section{MYL9 is much higher in PDNSC compared with that in paratumor tissues}

We detected the levels of MYL9 mRNA in 20 paired of PDNSC and paratumor tissues using RT-qPCR, and also detected the protein of MYL9 in SUNE-1 and HONE-1 cell lines (Fig. 1C). Compared with the control tissues, MYL9 was upregulated in PDNSC ( $<<0.001$, Fig. 1A, B). 


\section{MYL9 knockdown inhibits PDNSC cell proliferation in vitro}

To explore the function of MYL9 in PDNSC cell carcinogenesis, siMYL9 was used to transfect PDNSC SUNE-1 and HONE-1 cells, and the knockdown effects were both significant (Fig. 2A). MTT and EdU assays showed that MYL9 knockdown significantly decreased the proliferation of SUNE-1 and HONE-1 cells (Fig. 2B, C).

\section{MYL9 knockdown inhibits PDNSC cell migration and invasion in vitro}

To explore the function of MYL9 in PDNSC SUNE-1 and HONE-1 cells progression, Transwell and Boyden assays were performed. And the results showed that MYL9 knockdown significantly decreased the migration and invasion of SUNE-1 and HONE-1 cells (Fig. 2D, E).

\section{MYL9 knockdown reduces the $\mathrm{IC}_{50}$ value of paclitaxel in PDNSC cells in vitro}

Drug sensitivity test showed that the $\mathrm{IC}_{50}$ value of MYL9 knockdown cells was statistically lower than those of the controls (Fig. 2F).

\section{MYL9 knockdown inhibits PDNSC cell carcinogenesis, progression and chemoresistance via EMT signals}

To further explore the mechanism of MYL9 in PDNSC cells, Western blot was performed to detect the role of MYL9 in regulating EMT signals. And the results showed that EMT signals and its downstream chemoresistant factors (including $A B C B 1$ and $A B C G 2$ ) were significantly downregulated in MYL9 knockdown group (Fig. 3A). Therefore, MYL9 promoted the proliferation, progression and paclitaxel chemoresistance of PDNSC in vitro via EMT signals.

\section{MYL9 knockdown inhibits the mRNA expression of ABCB1}

Interestingly, we found that MYL9 knockdown (siMYL9) could inhibit the mRNA expression of ABCB1, which showes MYL9 may regulate ABCB1 mRNA expression (Fig. 3B).

\section{Discussion}

Several reports show that MYL9 expression is downregulated compared with the corresponding normal tissue $^{[11-15]}$ in bladder, colon, non-small cell lung, gastric, prostate cancers. However, MYL9 is upregulated 
in ovarian cancer, esophageal squamous cell carcinoma and glioblastoma ${ }^{[7,10,27]}$. In this study, we demonstrated for the first time that MYL9 expression was much higher in PDNSC compared with that in paratumor tissues, which shows that MYL9 may exhibit a candidate oncogenic role in PDNSC.

In previous studies, MYL9 has been implicated in many physiological and pathological functions including cell proliferation, migration and invasion, and it plays an important role in human cancers ${ }^{[6-12]}$. To further validate the exact role of MYL9 in PDNSC, we conducted siMYL9 to SUNE-1 and HONE-1 cell lines, and the results showed that MYL9 knockdown suppressed the cell proliferation, migration and invasion using in vitro assays. Earlier reports showed that MYL9 serves a tumor suppressor role in gastric cancer, colon cancer ${ }^{[12,14]}$. Inversely, recent reports ${ }^{[7,10,11]}$ show that MYL9 serves an oncogenic role, such as melanoma, glioblastoma and breast cancer. In this study, we demonstrated for the first time that MYL9 serves an oncogenic role in PDNSC and promotes PDNSC cell proliferation, migration and invasion.

Epithelial-mesenchymal transition (EMT) is a process in which epithelial cells acquire mesenchymal features ${ }^{[28]}$, and it is associated with tumor initiation, invasion, metastasis, and resistance to therapy ${ }^{[29,30]}$. EMT has been demonstrated to be the crucial signal in the proliferation, migration, invasion and paclitaxel chemoresistance of various cancers ${ }^{[31-34]}$. In the present study, our results showed for the first time that MYL9 knockdown suppressed N-cadherin, Vimentin, but increased the expression of Ecadherin. So our results showed that MYL9 knockdown inhibited PDNSC cell migration, invasion and increased paclitaxel sensitivity in vitro via the EMT signals, which is consistent with the previous studies.

Recently, more and more studies have confirmed that $A B C B 1$ and ABCG2 are the downstream signals of $\mathrm{EMT}^{[35]}$. ABCB1 and ABCG2 are the classic chemoresistant factors of cancer cells ${ }^{[36]}$. The results of the present study showed that the expression levels of $A B C B 1$ and $A B C G 2$ protein in PDNSC cells were reduced after MYL9 gene expression was inhibited, and also MYL9 knockdown inbibited the mRNA expression of $A B C B 1$, which showed that MYL9 might regulate $A B C B 1$ expression, and then to promote paclitaxel resistance in vitro. In future, we need more in vivo and in vitro experiments to demonstrate its detailed molecular mechanism.

Taken together, the results reveal that MYL9 is upregulated in PDNSC tissues compared with that in paratumor tissues, and MYL9 knockdown suppresses PDNSC cell proliferation, migration, invasion and paclitaxel resistance via EMT signals and downstream chemoresistant factors (including ABCB1 and ABCG2). Thus, we conclude that MYL9 serves as an oncogenic gene in PDNSC, which can be a useful patent of candidate treatment target biomarker in PDNSC.

\section{Conclusion}

In conclusion, the present study demonstrated that MYL9 is upregulated in PDNSC tissues, and MYL9 can promote PDNSC cell proliferation, migration, invasion and paclitaxel chemoresistance via EMT signals, and it may be used as a useful patent of therapeutic target in PDNSC. 


\section{Current \& Future Developments}

In future, more studies such as in vivo experiments are required to demonstrate the exact role of MYL9 and its molecular mechanism of action behind the carcinogenesis, progression and paclitaxel chemoresistance of PDNSC.

\section{Declarations}

\section{Ethics approval and consent to participate}

The Ethics Committee of The Integrated Hospital of Traditional Chinese Medicine, Southern Medical University authorized the experimental and research protocols of this study. All procedures performed in this study were according with the ethical standards of the institutional research committee and with the 1964 Helsinki declaration and its later amendments or comparable ethical standards. Written informed consent was provided and signed by all patients prior to sample collection.

\section{Consent for publication}

Not applicable.

\section{Availability of Data and Materials}

There are no DNA or RNA or Protein sequencing data in this study. And all of the datasets generated and/or analysed during the current study are available in zengruifang2008@163.com.

\section{Competing interests}

The authors declare no potential conflicts of interest.

\section{Funding}

This study was supported by the Guangdong Basic and Applied Basic Research Foundation (grant no. 2020A1515110030).

\section{Authors' Contributions}

ZRF performed the experiments and writed the original draft, WHY and QYH participated in the experiments and did the data curation with software, ZLS participated in the experiments and provided 
the resources, LR conceived, designed, supervised the experiments and provided the funding acquisition. All authors read and approved the final manuscript.

\section{Acknowledgements}

We gratefully thank Dr. Yunhui Qiu for her help in patients' samples collection of nasopharyngeal carcinoma.

\section{References}

1. Chen YP, Chan ATC, Le QT, et al. Nasopharyngeal carcinoma. Lancet 2019;394(10192):64-80.

2. Chua MLK, Wee JTS, Hui EP, et al. Nasopharyngeal carcinoma. Lancet 2016;387(10022):1012-1024.

3. Lee HM, Okuda KS, González FE, et al. Current Perspectives on Nasopharyngeal Carcinoma. Adv Exp Med Biol 2019;1164:11-34.

4. Claude $L$, Jouglar $E$, Duverge $L$, et al. Update in pediatric nasopharyngeal undifferentiated carcinoma. Br J Radiol 2019;92(1102):20190107.

5. Tu C, Zeng Z, Qi P, et al. Identification of genomic alterations in nasopharyngeal carcinoma and nasopharyngeal carcinoma-derived Epstein-Barr virus by whole-genome sequencing. Carcinogenesis 2018 31;39(12):1517-1528.

6. Zhu K, Wang Y, Liu L, Li S, Yu W. Long non-coding RNA MBNL1-AS1 regulates proliferation, migration, and invasion of cancer stem cells in colon cancer by interacting with MYL9 via sponging microRNA412-3p. Clin Res Hepatol Gastroenterol. 2020 Feb;44(1):101-114.

7. Wang JH, Zhang L, Huang ST, Xu J, Zhou Y, Yu XJ, Luo RZ, Wen ZS, Jia WH, Zheng M. Expression and prognostic significance of MYL9 in esophageal squamous cell carcinoma. PLoS One. 2017 Apr 7;12(4):e0175280.

8. Zhao B, Baloch Z, Ma Y, Wan Z, Huo Y, Li F, Zhao Y. Identification of Potential Key Genes and Pathways in Early-Onset Colorectal Cancer Through Bioinformatics Analysis. Cancer Control. 2019 Jan-Dec;26(1):1073274819831260.

9. Kim JS, Kim B, Lee HK, Kim HS, Park EJ, Choi YJ, Ahn GB, Yun J, Hong JT, Kim Y, Han SB. Characterization of morphological changes of B16 melanoma cells under natural killer cell attack. Int Immunopharmacol. 2019 Feb;67:366-371.

10. Kruthika BS, Sugur H, Nandaki K, Arimappamagan A, Paturu K, Santosh V. Expression pattern and prognostic significance of myosin light chain 9 (MYL9): a novel biomarker in glioblastoma. J Clin Pathol. 2019 Oct;72(10):677-681.

11. He H, Wang D, Yao H, Wei Z, Lai Y, Hu J, Liu X, Wang Y, Zhou H, Wang N, Luo XG, Zhang TC.

Transcriptional factors p300 and MRTF-A synergistically enhance the expression of migration-related genes in MCF-7 breast cancer cells. Biochem Biophys Res Commun. 2015 Nov 27;467(4):813-20. 
12. Gu W, Ren JH, Zheng X, Hu XY, Hu MJ. Comprehensive analysis of expression profiles of long noncoding RNAs with associated ceRNAnetwork involved in gastric cancer progression. Mol Med Rep. 2019 Sep;20(3):2209-2218

13. Lu Y, Liu P, Wen W, et al. Cross-species comparison of orthologous gene expression in human bladder cancer and carcinogen-induced rodent models. Am J Transl Res. 2010;3(1):8-27.

14. Yan Z, Li J, Xiong Y, et al. Identification of candidate colon cancer biomarkers by applying a random forest approach on microarray data. Oncol Rep. 2012;28:1036-42.

15. Tan $X$, Chen M. MYLK and MYL9 expression in non-small cell lung cancer identified by bioinformatics analysis of public expression data. Tumor Biol. 2014;35:12189-200.

16. Huang YQ, Han ZD, Liang YX, et al. Decreased expression of myosin light chain MYL9 in stroma predicts malignant progression and poor biochemical recurrence-free survival in prostate cancer. Med Oncol 2014;31.

17. Chua ML, Sun Y, Supiot S. Advances in nasopharyngeal carcinoma-"West meets East". Br J Radiol 2019;92(1102):20199004.

18. Lee AWM, Ng WT, Chan JYW, et al. Management of locally recurrent nasopharyngeal carcinoma. Cancer Treat Rev 2019;79:101890.

19. Ng WT, Corry J, Langendijk JA, et al. Current management of stage IV nasopharyngeal carcinoma without distant metastasis. Cancer Treat Rev 2020;85:101995.

20. Kheifetz, et al.Method and system for prediction of medical treatment effect.US 20170140109 A1, 2017.

21. Lewis, et al.Compounds, compositions and methods for cancer treatment.US 20200062741 A1, 2020.

22. Guan S, Wei J, Huang L, et al. Chemotherapy and chemo-resistance in nasopharyngeal carcinoma. Eur J Med Chem 2020 1;207:112758.

23. Cui Z, Pu T, Zhang Y, et al. Long non-coding RNA LINC00346 contributes to cisplatin resistance in nasopharyngeal carcinoma by repressing miR-342-5p. Open Biol. 2020;10(5):190286.

24. Wang X, Masters JR, Wong YC, et al. Mechanism of differential sensitivity to cisplatin in nasopharyngeal carcinoma cells. Anticancer Res. 2001;21(1A):403-8. Erratum in: Anticancer Res. 2005 Jan-Feb;25(1b):681. PMID: 11299769.

25. Liu S, Huang J, Zhang Y, Liu Y, Zuo S, Li R. MAP2K4 interacts with Vimentin to activate the PI3K/AKT pathway and promotes breast cancer pathogenesis.Aging (Albany NY). 2019;11(22):10697-10710.

26. Liu Y, Jiang Q, Liu X, et al. Cinobufotalin powerfully reversed EBV-miR-BART22-induced cisplatin resistance via stimulating MAP2K4 to antagonize non-muscle myosin heavy chain IIA/glycogen synthase $3 \beta / \beta$-catenin signaling pathway. EBioMedicine. 2019; 48: 386-404.

27. Deng Y, Liu L, Feng W, et al. High Expression of MYL9 Indicates Poor Clinical Prognosis of Epithelial Ovarian Cancer. Recent Pat Anticancer Drug Discov.2021; Jul 6. doi: $10.2174 / 1574891 \times 16666210706153740$. Online ahead of print. 
28. Sinha D, Saha P, Samanta A, Bishayee A. Emerging Concepts of Hybrid Epithelial-to-Mesenchymal Transition in Cancer Progression.Biomolecules. 2020;10(11):1561. Published 2020 Nov 16.

29. Bakir B, Chiarella AM, Pitarresi JR, Rustgi AK. EMT, MET, Plasticity, and Tumor Metastasis. Trends Cell Biol. 2020;30(10):764-776.

30. Bakir B, Chiarella AM, Pitarresi JR, Rustgi AK. EMT, MET, Plasticity, and Tumor Metastasis. Trends Cell Biol. 2020;30(10):764-776.

31. Dudas J, Ladanyi A, Ingruber J, Steinbichler TB, Riechelmann H. Epithelial to Mesenchymal Transition: A Mechanism that Fuels Cancer Radio/Chemoresistance.Cells. 2020;9(2):428. Published 2020 Feb 12.

32. Wang S, Huang S, Sun YL. Epithelial-Mesenchymal Transition in Pancreatic Cancer: A Review.Biomed Res Int. 2017;2017:2646148.

33. Shibue T, Weinberg RA. EMT, CSCs, and drug resistance: the mechanistic link and clinical implications.Nat Rev Clin Oncol. 2017;14(10):611-629.

34. Ashrafizadeh M, Mirzaei S, Hashemi F, Zarrabi A, Zabolian A, Saleki H, Sharifzadeh SO, Soleymani L, Daneshi S, Hushmandi K, Khan H, Kumar AP, Aref AR, Samarghandian S. New insight towards development of paclitaxel and docetaxel resistance in cancer cells: EMT as a novel molecular mechanism and therapeutic possibilities. Biomed Pharmacother. 2021 Sep;141:111824.

35. De Las Rivas J, Brozovic A, Izraely S, Casas-Pais A, Witz IP, Figueroa A. Cancer drug resistance induced by EMT:novel therapeutic strategies.Arch Toxicol. 2021;95(7):2279-2297.

36. Wu X, Zhao J, Ruan Y, Sun L, Xu C, Jiang H. Sialyltransferase ST3GAL1 promotes cell migration, invasion, and TGF- $\beta 1$-induced EMT and confers paclitaxel resistance in ovarian cancer. Cell Death Dis. 2018 Oct 30;9(11):1102.

\section{Figures}




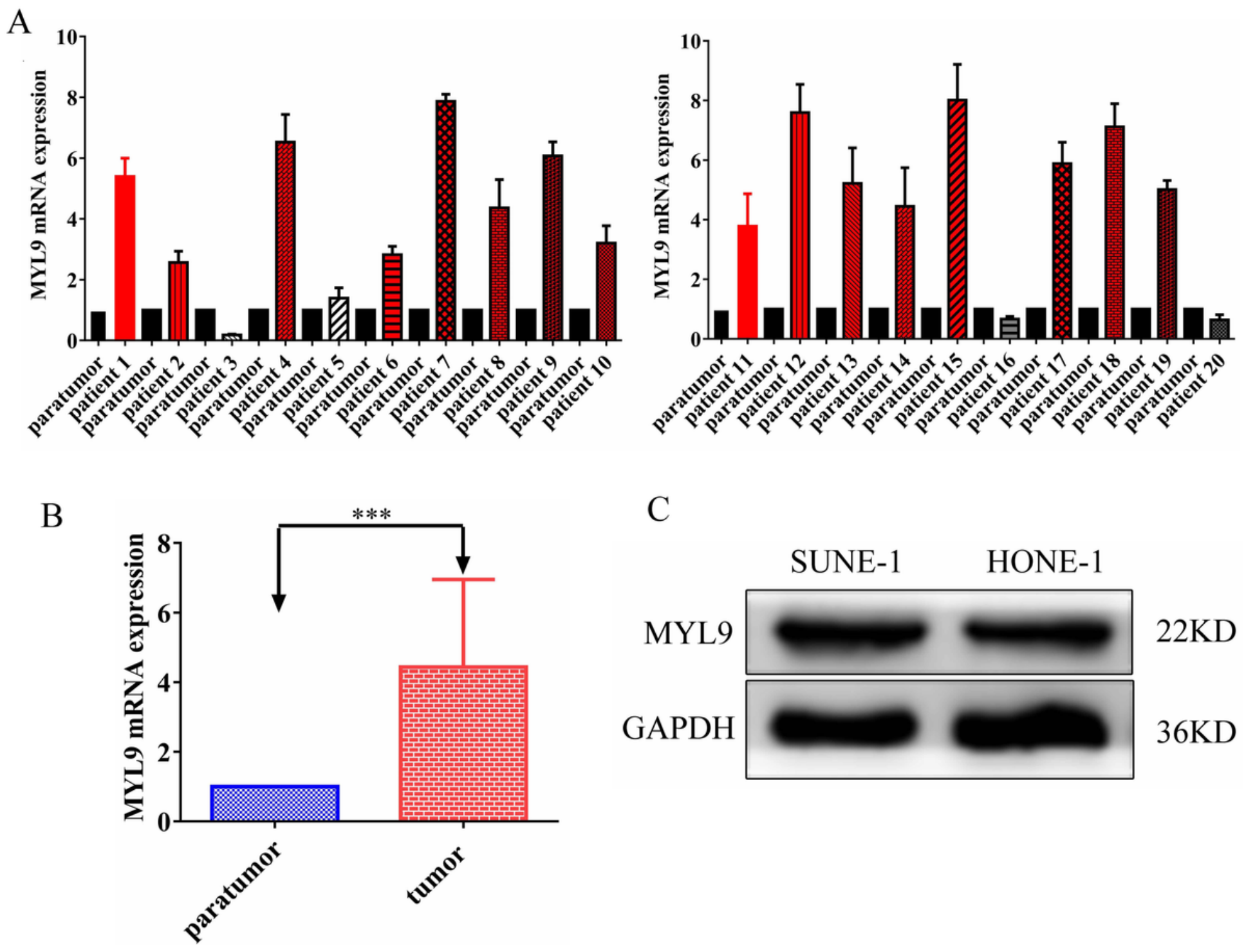

Figure 1

MYL9 is found to be highly expressed in PDNSC using RT-qPCR assay.

A and B. MYL9 mRNA expression in PDNSC and paratumor tissues $(\mathrm{P}<0.001)$. C. The expression of MYL9 protein in PDNSC cell lines. 
A

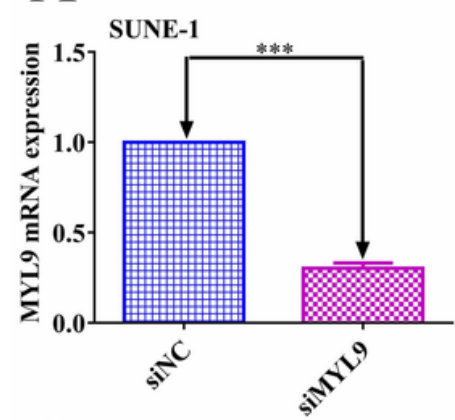

$\mathrm{C}$

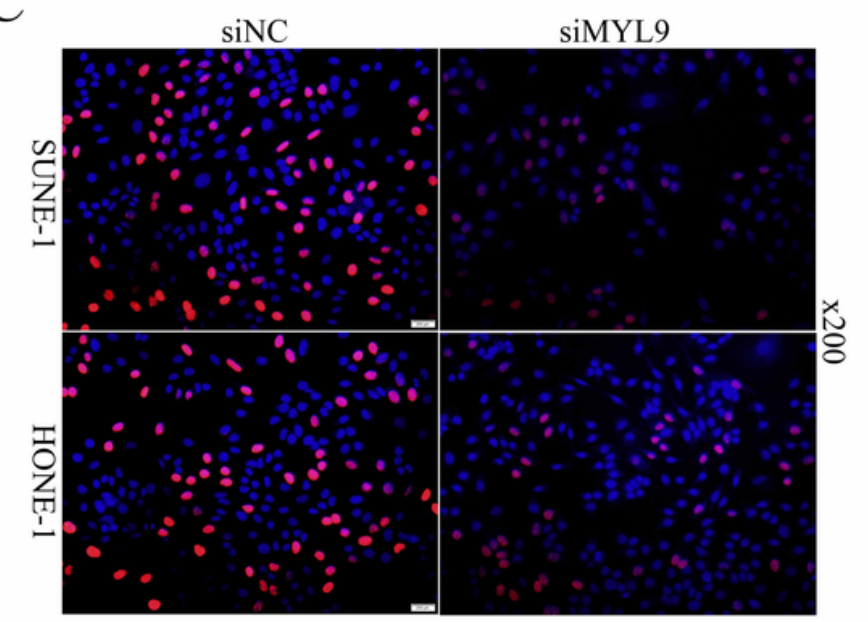

$\mathrm{E}$

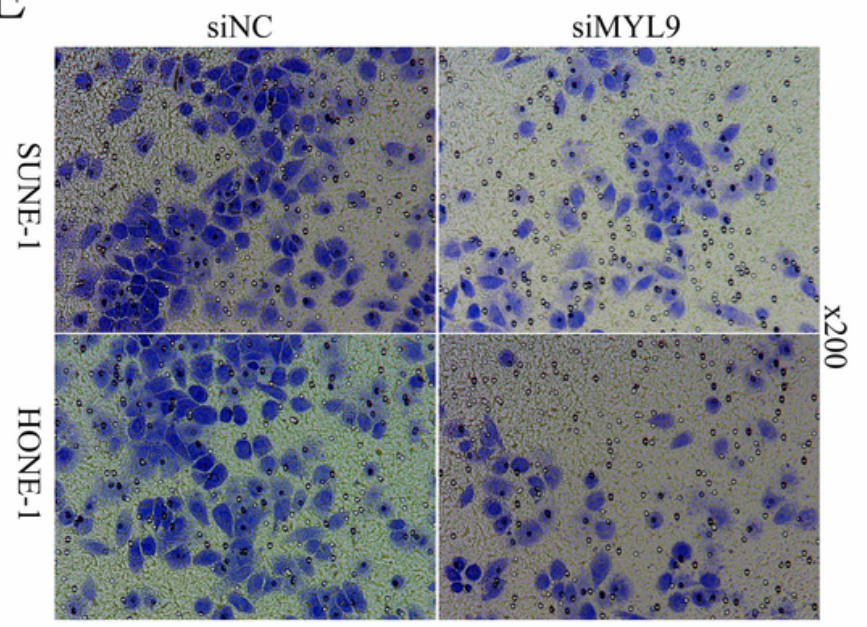

SiMYL9
B

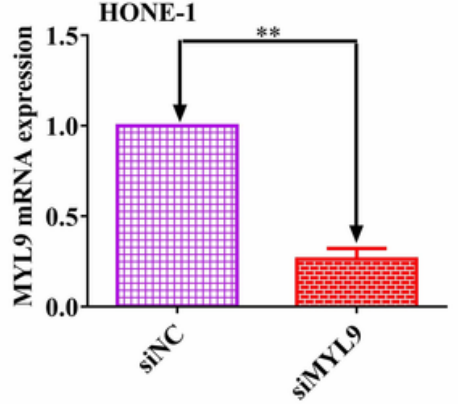

$\mathrm{F}$
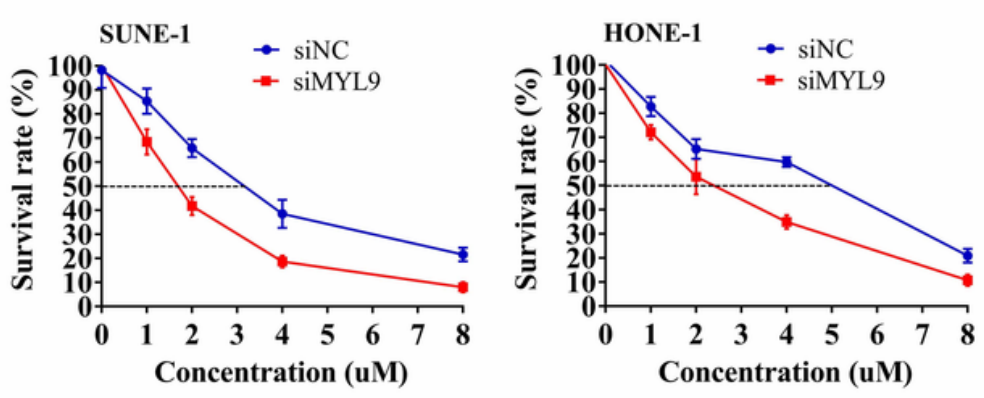

Figure 2

MYL9 Knockdown suppresses PDNSC cell proliferation, migration, invasion and paclitaxel chemoresistance

A. The knockdown effects of siRNA targeting MYH9 in PDNSC SUNE-1 and HONE-1 cells. B. Knocking down PDNSC suppressed cell proliferation identified by MTT assay in PDNSC SUNE-1 and HONE-1 cells. C. Knocking down MYL9 reduced cell proliferation identified by EdU in PDNSC cells ( $x 200$ magnification; scale bar, $50 \mu \mathrm{m})$. D. Knocking down MYL9 reduced cell migration identified by Transwell assay in PDNSC cells (x200 magnification; scale bar, $50 \mu \mathrm{m}$ ). E. Knocking down MYL9 reduced cell invasion 
identified by Boyden assay in PDNSC cells (x200 magnification; scale bar, $50 \mu \mathrm{m}$ ). F. MYL9 knockdown reduces the IC50 value of paclitaxel in PDNSC cells in vitro.

A

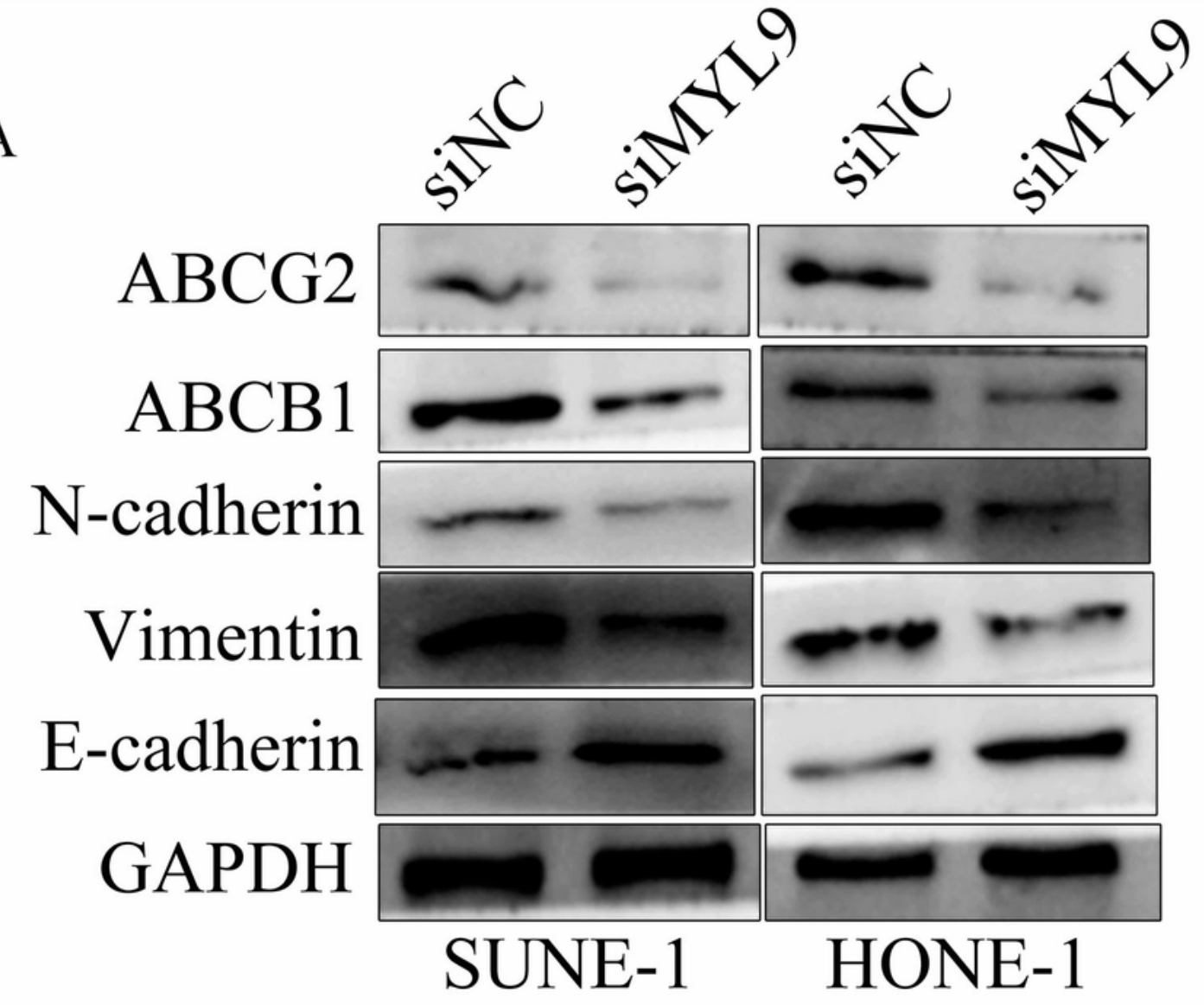

B

SUNE-1

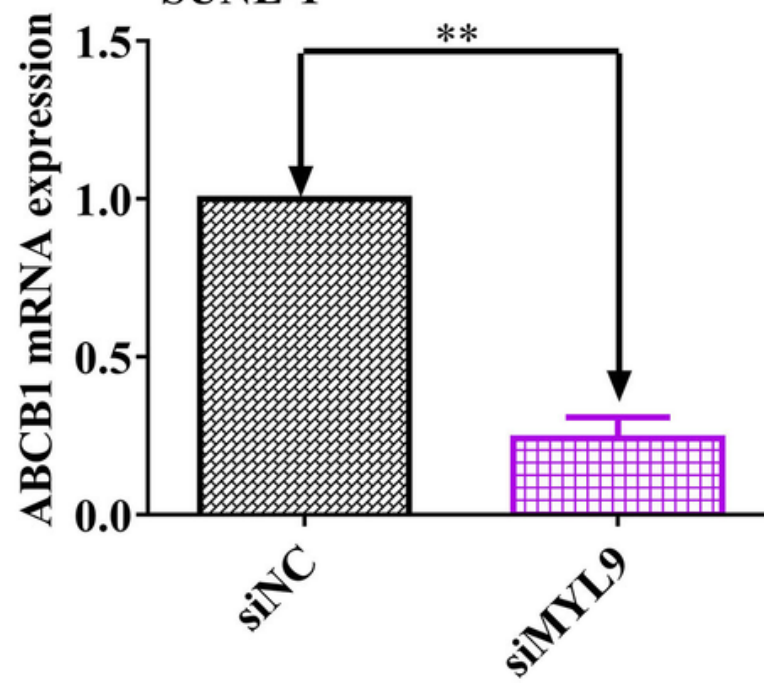

HONE-1

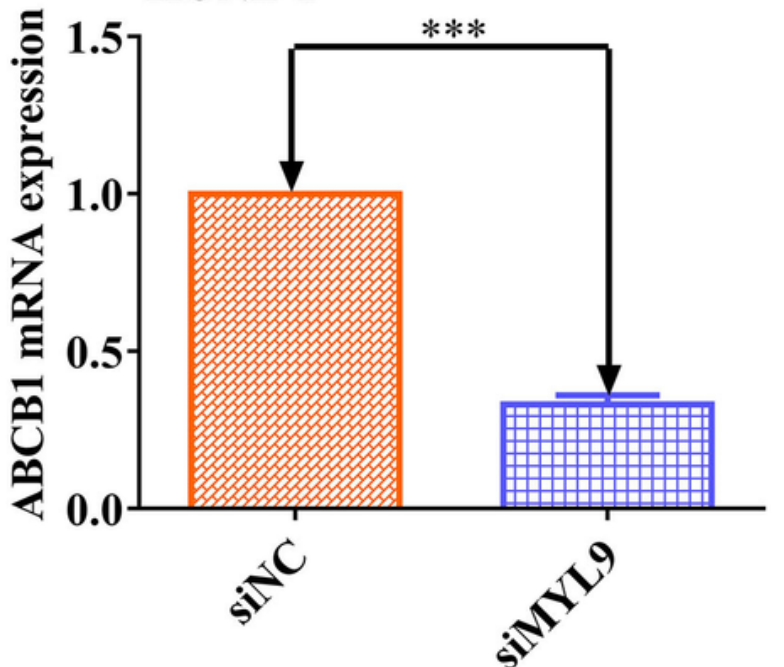

Figure 3

MYH9 knockdown inhibits the EMT signals using Western blot analysis, and also inhibits ABCB1 mRNA expression using RT-qPCR analysis. 


\section{Supplementary Files}

This is a list of supplementary files associated with this preprint. Click to download.

- WBoriginalplots.pdf 